\title{
MICROSURGICAL LYMPHATIC VASCULAR GRAFTING AND SECONDARY LIPOSUCTION: RESULTS OF COMBINATION TREATMIENT IN SECONDARY LYMPHEDEMA
}

R.G.H. Baumeister, J. Wallmichrath, M. Weiss, S.H.C. Baumeister, A. Frick

Consultant in Lymphology and Microsurgery Chirurgische Klinik Muenchen Bogenhausen and Urologische Klinik Muenchen Planegg, Munich; Former Head of the Division of Plastic-, Hand- and Microsurgery, Department of Surgery (JW), Campus Grosshadern, Ludwig-Maximilians University, Munich; Division of Hand, Plastic and Aesthetic Surgery (JW,AF), and Department of Nuclear Medicine (MW), University Hospital, LMU Munich, Germany; and Division of Pediatric Oncology, Dana-Farber Cancer Institute, and Division of Pediatric Hematology-Oncology, Boston Children's Hospital and Harvard Medical School (SB), Boston, MA, USA

\section{ABSTRACT}

Surgical treatment of lymphedema with liposuction typically requires subsequent compression therapy. Here we describe an approach where secondary arm lymphedemas are initially treated by autologous lymphatic grafting to bypass the axilla and restore lymphatic flow. In the presence of excess adipose tissue, liposuction is then performed in a second procedure. To assess outcomes, the authors evaluated 28 consecutive adult patients who had undergone secondary liposuction following lymphatic grafting. Arm volumes were measured prior to lymphatic grafting and after the secondary liposuction. The necessity for additional treatment by compression garment and manual lymphatic drainage was assessed prior to lymphatic grafting and after the secondary liposuction following the direct postoperative regimen. The mean arm volumes were reduced significantly $(p<0.001)$ from a mean of $3417 \pm 171(S E M) \mathrm{cm}^{3}$ prior to lymphatic grafting to $3020 \pm 125 \mathrm{~cm}^{3}$ after reconstruction of the lymphatic vascular system and finally to $2516 \pm 104 \mathrm{~cm}^{3}$ after the secondary liposuction (SLS). All 28 adult patients underwent contin- uous compression and manual lymph-drainage (MLD) prior to the reconstructive surgery. All 28 patients were evaluated regarding necessity of any additional therapy more than 6 months after SLS with a median follow up period of 37 months (range, 7-160 months). 18 of 28 patients did not require any supportive therapy beyond 6 months after SLS to maintain the results. Three patients continued to utilize manual lymphatic drainage, 4 used a combination of MLD and compression therapy and 3 used elastic compression therapy (one patient only while at work). These results indicate that microsurgical restoration of lymphatic outflow followed by SLS eliminates the need for additional treatment in more than two thirds of patients.

Keywords: lymphedema, lymphatic vessel grafting, liposuction, combination therapy

Arm edemas after oncological treatment of mammary carcinomas are the most prevalent lymphedemas in Western countries. Complete Decongestive Therapy (CDT) consisting of Manual Lymph Drainage (MLD), Decongestive Kinesiotherapy, and Compres- 
sion Therapy is the standard treatment. However, there is an increasing interest in surgical procedures for the treatment of lymphedemas. Modern resection methods using suction devices and microsurgical procedures are increasingly used but little is reported about additional treatments required after surgery. In our studies assessing quality of life (1), we found that use of compression garments represents a significant burden for patients. Furthermore, MLD sessions are often time consuming and impact patients' quality of life.

Autologous lymphatic vessel grafting overcomes a locally compromised lymphatic pathway. The autologous lymphatic vessels are harvested from the thigh where there is a surplus of lymphatic collectors and are subsequently anastomosed with the main lymphatic collectors proximally and distally to the blockage serving as a bypass (2-4) (Fig. 1). Lymphatic scintigraphy studies demonstrated that this method leads to a significant increase in lymphatic fluid transport capacity through the grafts $(5,6)$. In patients with visualization of the grafts as bundles of activity, normal values can be reached (7).

The problem of secondary tissue changes such as fibrosis and increased fat deposits, however, may not be solved with reconstruction of the fluid transport alone. After restoration of the lymphatic vascular system, resection of these tissue compounds may be advisable to improve arm volumes and appearance of the limb. In these patients, the question also arises whether initial autologous lymphatic vessel grafting alters the necessity to wear elastic garments after liposuction in lymphedemas.

In this retrospective study, we examined whether reconstructive surgery using the patients' own lymphatic vessels followed by secondary liposuction eliminates the necessity for additional treatments, which may represent a significant burden on quality of life.

\section{MATERIALS AND METHODS}

28 adult patients who were originally treated with autologous lymphatic grafts for arm lymphedemas due to a locally obstructed lymphatic system underwent liposuction as a secondary procedure. Lymphatic vessel grafting is an officially accepted surgical approach for the treatment of lymphedemas in Germany and has been previously described in further detail $(2-4,8)$. This retrospective study was part of the hospital's standard quality management procedure and deemed exempt from additional IRB approval. All data were deidentified.

Briefly, the autologous lymphatic vessels are harvested from the thigh where there is a surplus of lymphatic collectors. Prior to the lymphatic vessel harvest, lymphatic scintigraphy is performed in each patient to exclude the possibility of an underdeveloped lymphatic system dependent on the existing lymphatic vasculature (asymptomatic lymphatic insufficiency). Similarly, the presumed location of the lymphatic blockage proximally to the lymphedema within the area of prior axillary lymph node dissection is confirmed by lymphoscintigraphy. Intraoperatively, a dye (Patent blue ${ }^{\circledR}$ ) is injected subdermally in the first and second webspace between the toes. The lymphatic collectors of the ventro-medial bundle (which contains about 16 collectors) at the thigh are thereby stained within approximately 15 minutes. About 2 to 3 collectors (at a length of about 23 to 30 centimeters) are harvested only if a sufficient amount of other stained lymphatic vessels can be left in place. A suture is placed on each proximal end of the collector graft and a long thread kept in place and used for pulling the graft. Distally each graft is left open. The proximal end of the distal remaining lymphatic vessel is sealed using a suture or cautery to avoid formation of fistulas or uninhibited lymphatic drainage into the tissue. The distal end of the proximal remaining lymphatic vessel is left open.

In the upper arm, a short oblique superficial skin incision is made and lymphatic collectors (which are often fibrotic in advanced stages) in the subcutaneous tissue of the lymphedema are identified using the operating 
microscope. The distal anastomosis typically involves two to four main lymphatic collectors with a diameter of about $0.3 \mathrm{~mm}$. Similarly, a skin incision is made 2-3 centimeters above the clavicle and lymphatic collectors, and lymph nodes at the neck are identified beneath the sternocleidomastoid muscle lateral to the internal jugular vein. When the proximal anastomosis is made to a lymph node, the capsule is fenestrated, and the graft attached with single sutures to ensure a direct connection to the marginal sinuses within the lymph node. Between the two skin incisions, a drainage tube is placed subcutaneously and flushed with Lactated Ringer's solution to moisturize the tube and minimize friction. Subsequently the lymphatic grafts are threaded through the tube from the arm to the neck to minimize friction or injury to the graft during insertion.

The tube is then pulled out distally. After the tube is removed and the lymphatic grafts are in position, they are subsequently anastomosed with the main lymphatic collectors distally and lymphatic collectors or lymph nodes proximally to the blockage serving as a bypass (Fig. 1). High power microscopes are required for suturing the lympho-lymphatic anastomosis. It is critical to perform the anastomosis without tension in oblique direction using absorbable suture material. The size of the lymphatic vessels, instrumentation, sutures and magnification levels are as previously described $(2-4,8)$.

In patients with a predominance of fat tissue, in whom an additional reduction of arm volumes was desired, liposuction was performed as a secondary procedure. The suction was performed in a longitudinal direction in order to spare the lymphatic main collectors as described by Frick et al (9) and Hofmann et al (10). The suction was performed only beneath the area of the lymphatic anastomosis at the proximal upper arm.

Most patients included in this study suffered from sequelae of mammary carcinoma treatment. The mean age at secondary liposuction was 56.3 years (range, 41.6 - 72.4 years). The median interval between lymphatic grafting and liposuction was 21 months (range 9-107 months).

Prior to lymphatic grafting, all patients completed a non-operative treatment regimen of CDT for at least 6 months. Most patients participated in non-operative treatment regimens for years before presenting for lymphatic grafting. After lymphatic vessel grafting and secondary liposuction, a flat knitted compression garment was used routinely for 6 months as a standard postoperative regimen to initially support the influx of lymph into the lymphatic anastomoses and stabilize the tissues after the secondary liposuction respectively. Two garments were prescribed and continuous use ( 24 hours/day) recommended initially. No manual lymph drainage was performed during this time period.

After 6 months, it was determined whether the patient was able to eliminate all additional therapeutic measures or if an additional treatment with compression therapy or manual lymph drainage was deemed necessary to maintain the result. In most patients, this decision was made during standard clinical follow-up visits at our center. If this was not possible for patient-based reasons, the decision was made after consultation with the patient's primary care physician and in telephone interviews. Only patients with additional follow-up extending beyond the 6 months after surgery were included in the study. The use of additional therapies was explored and documented during routine follow-up consultations or by phone interviews within the standard clinical follow-up period.

Arm volumes were determined measuring the circumference with an interval of $4 \mathrm{~cm}$ and using the truncated cone formula according to Kuhnke (11).

\section{Statistical Analysis}

The statistical analysis was performed by SPSS. A p-value of $\leq \mathbf{0 . 0 5}$ was considered significant. For the statistical analysis of differences in arm volume, the nonparametric Wilcoxon signed-rank test was used to 
compare samples at different time points. The non-parametric analysis of the necessity of garment or MLD use before and after operative treatment was performed using $\mathrm{McNe}$ mar's test for paired samples.

\section{RESULTS}

Of 215 patients with arm edemas treated between 1980 and 2014 by autologous lymphatic grafting, 28 adult patients who had undergone autologous lymphatic grafting at very advanced stages of lymphedema also underwent SLS as a second procedure. As detailed in Methods, all patients had been pretreated extensively by non-operative means prior to lymphatic grafting, with a minimum pre-treatment period of 6 months.

After the first surgery for reconstruction of the interrupted lymphatic vascular system, arm volumes in the 28 adult patients decreased significantly from a mean of $3417 \pm 171 \mathrm{~cm}^{3}$ (SEM) to a mean of $3020 \pm 125 \mathrm{~cm}^{3}$ (SEM) $(\mathrm{p}<0.001)$. At this stage, 7 of the 28 patients used compression regularly, 15 under working conditions, and 9 patients maintained manual lymphatic drainage. This unusually high requirement for continued compression therapy or MLD following autologous lymphatic grafting reflects the advanced stages of lymphedema in this group of 28 patients.

After SLS, arm volumes further decreased from a mean of $3020 \pm 125 \mathrm{~cm}^{3}$ (SEM) to a mean of $2516 \pm 104 \mathrm{~cm}^{3}(\mathrm{SEM})(\mathrm{p}<0.001)$ with a median follow-up period of 37 months (range, 7-160 months) after SLS. The volume reduction achieved with this sequential surgical method resulted in a volume comparable

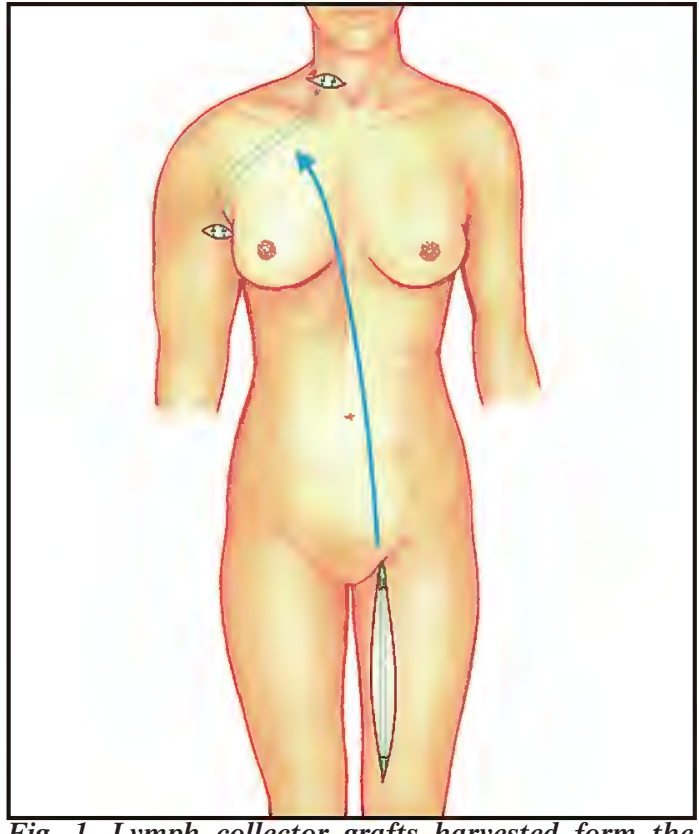

Fig. 1. Lymph collector grafts harvested form the thigh bypass a lymphatic obstruction at the axilla and now connect the lymphatic vascular system of the upper arm to the lymphatic system of the neck.

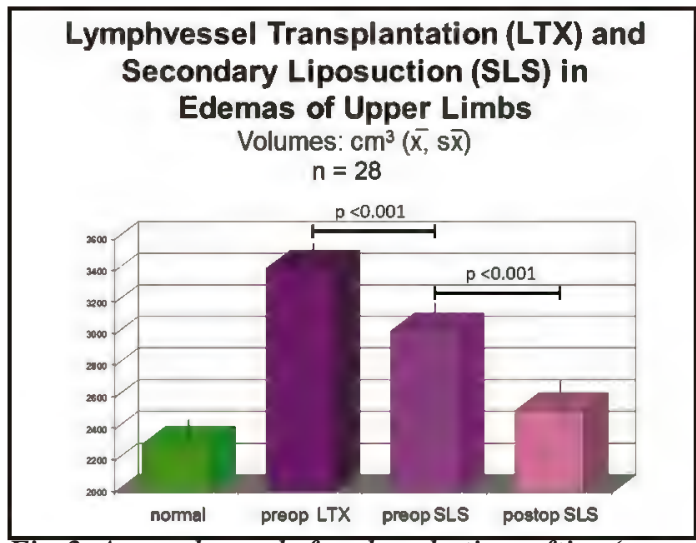

Fig. 2. Arm volumes before lymphatic grafting (preop LTX), after lymphatic grafting (preop SLS), and after secondary liposuction (postop SLS).

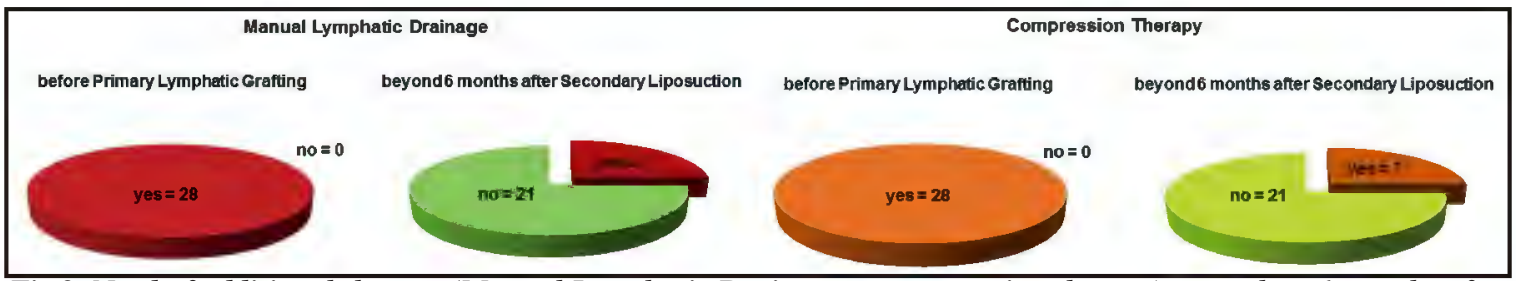

Fig.3. Need of additional therapy (Manual Lymphatic Drainage or compression therapy) more than 6 months after secondary liposuction (patients had previous lymphatic grafting). 
to that of the normal contralateral arm with a mean volume of $2304 \pm 79 \mathrm{~cm}^{3}$ (SEM) (Fig. 2) as measured at the time prior to lymphatic vessel grafting. The differences in affected arm volume between the preoperative status prior to the lymphatic grafting and the secondary liposuction compared to the status after the liposuction where highly significant. In contrast, control volumes of the contralateral arms did not change significantly over time with a mean volume of $2304 \pm 79 \mathrm{~cm}^{3}$ (SEM) measured prior to the microsurgical procedure and a mean volume of $2291 \pm 90(\mathrm{SEM}) \mathrm{cm}^{3}$ at the time of follow-up after SLS ( $p>0.05$ ).

Elastic compression therapy was continued as standard in all patients after surgery for 6 months to improve the influx into the anastomosed lymphatic collectors. Manual lymphatic drainage was not performed during the 6 months following surgery.

After the 6-month postoperative period, compression therapy was discontinued in all patients after a clinical assessment. Assessments to determine the need for additional treatment were initiated at regular intervals after this period. Whether patients utilized additional supportive treatments was determined and documented in all patients either during a follow-up visit or during additional phone interviews.

All 28 adult patients who underwent primary lymphatic grafting followed by secondary liposuction and discontinuation of postoperative elastic compression therapy 6 months after the second surgical procedure were evaluated with follow-up beyond 6 months after secondary liposuction (Fig. 3).

With a median follow-up of 37 months (range, 7-160 months), 21 of 28 patients did not require additional compression therapy beyond 6 months following the secondary liposuction. Of the 7 patients who required ongoing use beyond 6 months following secondary liposuction, 6 used the garment continuously, whereas one patient used compression therapy only as needed while performing heavy manual labor (Fig. 3).

Manual lymphatic drainage did not need to be performed in 21 patients after secondary liposuction. While none of the 28 patients underwent manual lymphatic drainage in the 6 months following secondary liposuction, 7 patients elected to resume manual lymphatic drainage at 6 months after the second surgery. Four of 7 patients combined it with the use of elastic support (Fig. 3).

In summary, 18 of 28 patients did not require any supportive therapy beyond 6 months following secondary liposuction, while 3 patients continued to utilize manual lymphatic drainage, 4 used a combination of manual lymphatic drainage and compression therapy, and 3 used elastic compression therapy.

There was a statistically significant difference in the requirement of additional supportive therapies after surgical intervention (consisting of lymphatic vessel grafting followed by secondary liposuction) when compared to the requirement prior to surgical intervention with respect to use of compression garments $(\mathrm{p}<0.05)$ and manual lymphatic drainage $(\mathrm{p}<0.05)$.

Two representative patients are shown in Figs. 4-8.

A 60-year-old female underwent breast conserving therapy of mammary carcinoma. Figs. 4, 5 and 6 depict sequential imaging of the arm at various stages of treatment for lymphedema. The arm is shown prior to the first surgery while using CDT consisting of compression garments, manual lymphatic drainage and physical therapy. The edematous arm had a volume of $2712 \mathrm{~cm}^{3}$ compared to the volume of the healthy contralateral arm measuring $2045 \mathrm{~cm}^{3}$ (Figs. 4a,b). At 1 year following lymphatic grafting, the volume of the edematous arm had decreased to $2252 \mathrm{~cm}^{3}$, whereas the volume of the healthy arm remained relatively unchanged at $2073 \mathrm{~cm}^{3}$ (Figs. 5a,b). Following secondary liposuction (here shown at 6 years after secondary liposuction), the previously edematous arm had a volume of $1858 \mathrm{~cm}^{3}$ compared to $2200 \mathrm{~cm}^{3}$ of the healthy arm (Figs. 6a,b). This patient did not use any additional supportive therapy beyond 6 months following SLS. 


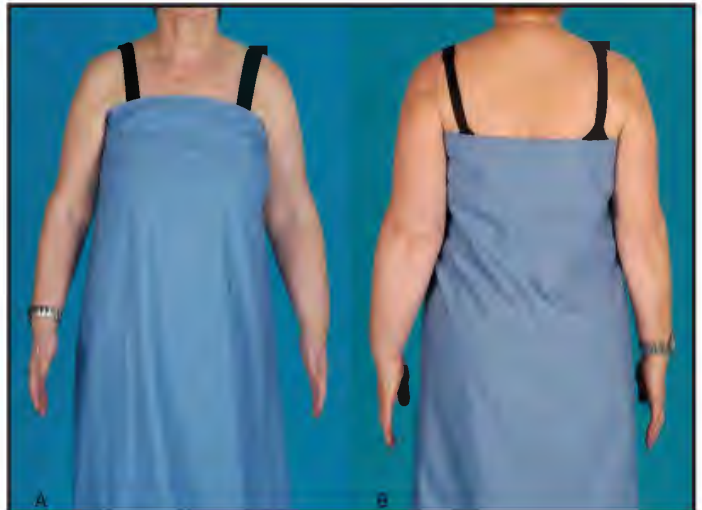

Fig. 4. A 60-year-old patient 30 months after breast conserving therapy, prior to lymphatic grafting. Arm volumes: $2045 \mathrm{~cm}^{3}$ of the healthy right arm, $2712 \mathrm{~cm}^{3}$ of the affected left arm in front (a) and rear (b) view.

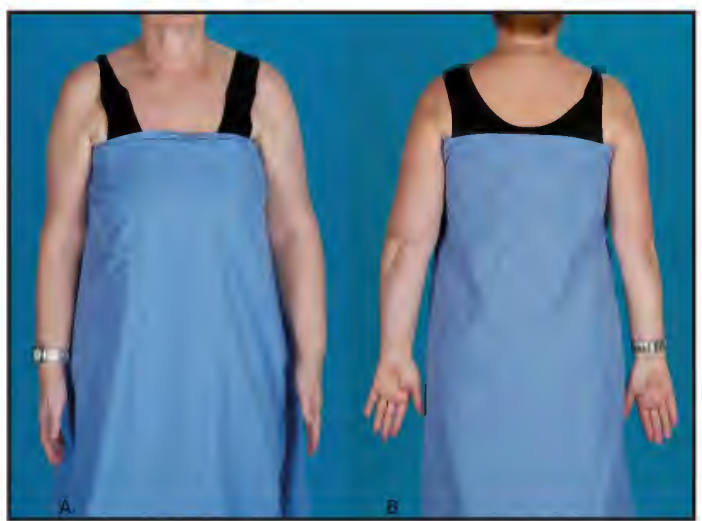

Fig. 5. The patient shown in Fig. one year after lymphatic grafting without additional therapy. Arm volumes: $2073 \mathrm{~cm}^{3}$ of the healthy right arm, $2252 \mathrm{~cm}^{3}$ of the affected left arm in front (a) and rear (b) view.

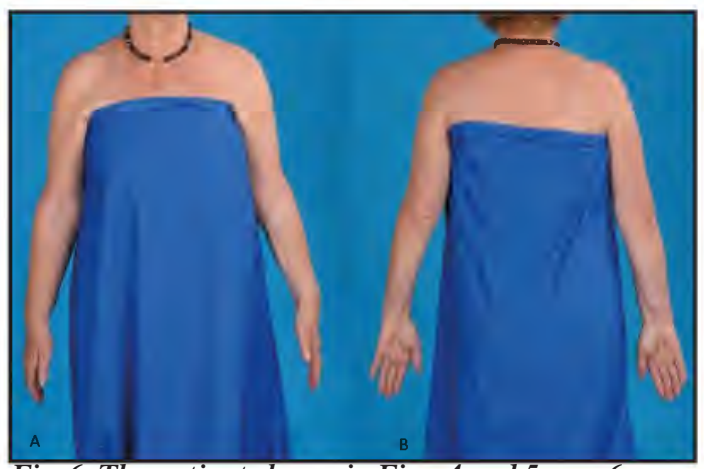

Fig. 6. The patient shown in Figs. 4 and 5 now 6 years after secondary liposuction without additional therapy. Arm volumes: $2200 \mathrm{~cm}^{3}$ of the healthy right arm, $1858 \mathrm{~cm}^{3}$ of the affected left arm in front (a) and rear (b) view.

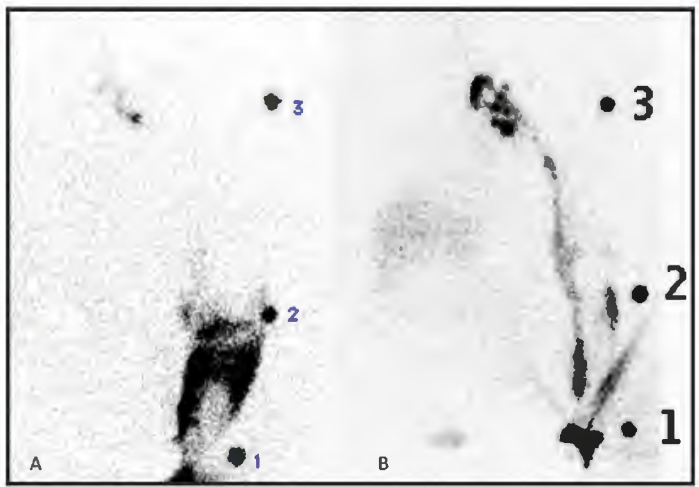

Fig. 7. Lymphatic transport in the patient shown in Fig. 4, 5 and 6 as demonstrated by lymphoscintigraphy of the right arm (marking points: 1 wrist, 2 elbow 3 shoulder). (a) Prior to lymphatic grafting 10 to 23 minutes after application of the radiopharmaceutical, imaging demonstrates accumulation of lymph at forearm without any flow to the axillary region. (b) 8 years after lymphatic grafting and 6 years after secondary liposuction, 10 to 25 minutes after injection of the radiopharmaceutical, imaging demonstrates quick outflow via lymphatic grafts to lymph nodes at the neck and absence of accumulation of lymph at the forearm.

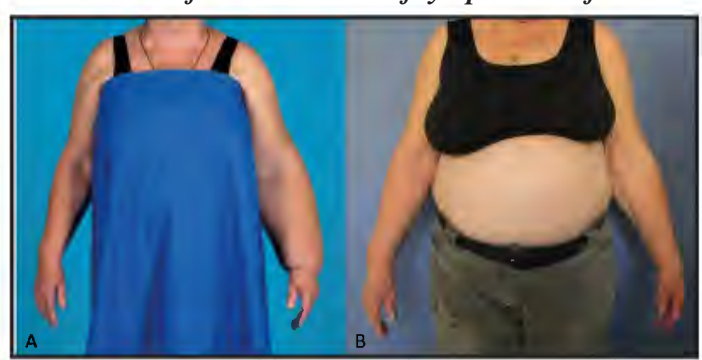

Fig. 8. A 64-year-old patient after treatment of mammary carcinoma with extensive lymphedema of the left arm. (a) Prior to lymphatic grafting. Arm volumes: $2965 \mathrm{~cm}^{3}$ of the healthy right arm, $6537 \mathrm{~cm}^{3}$ of the affected left arm. (b) 5 years after lymphatic grafting and 4 years after secondary liposuction without additional therapy. Arm Volumes: $3139 \mathrm{~cm}^{3}$ of the healthy right arm, $2529 \mathrm{~cm}^{3}$ of the affected left arm.

Lymphatic scintigraphy in the patient was performed prior to lymphatic grafting. The radio-pharmaceutical accumulated at the forearm indicating stasis of the lymphatic fluid. No flow to the axillary region was visible (Fig. 7a). 8 years after lymphatic grafting and 6 years after SLS, dramatic improvement in the lymphatic outflow can be seen, with visible activity along the lymphatic grafts and the supraclavicular lymph nodes. This scintigram result resembles a normal scintigram signal. 
The lymphatic transport index improved accordingly from a score of 21 (pathologic) to a score of 2 (normal) (Fig. 7b). For point of reference, a score of $\mathbf{1 0}$ is considered borderline.

A second representative patient is shown here prior to lymphatic grafting and during late follow-up after completing secondary liposuction (Fig. 8).

This 64-year-old female with excessive edema of the left arm after treatment of mammary carcinoma is shown while being treated with supportive therapy approaches prior to presenting for surgical care of her lymphedema. The edematous arm had a volume of $6537 \mathrm{~cm}^{3}$ compared to $2965 \mathrm{~cm}^{3}$ of the healthy arm (Fig.8a). Figure $8 \mathrm{~b}$ shows the treated left arm, which is now comparable to the right healthy arm in volume and appearance, without any additional treatment 5 years after lymphatic grafting and 4 years after SLS. The volume of the previously edematous arm decreased to $2529 \mathrm{~cm}^{3}$, whereas the normal arm showed a small increase to $3139 \mathrm{~cm}^{3}$.

\section{DISCUSSION}

Lymphedemas are caused by a diminished transport of lymphatic fluid (12). In the later stages of lymphedema, a surplus of adipose tissue and fibrosis is clinically obvious and further complicates successful therapy $(13,14)$. Both factors must be taken into consideration to address the problem comprehensively.

Addressing the diminished transport is basically a vascular problem. Lymphedemas due to a local obstruction, as can be seen in the axilla or the pelvis can be treated using lymphatic vascular bypasses. This approach is most applicable in secondary lymphedemas occurring after medical interventions in these areas. Rarely this is the case in primary lymphedemas of the lower extremity. Lymphatic grafting is only possible in unilateral primary lymphedemas arising from short regional atresia, in which a peripheral lymphatic system is intact, as was shown in lymphographic studies by Kinmonth (15). In leg lymphedemas, lymphatic vessels can be harvested and transferred from the healthy contralateral leg to bypass the regional atresia. In arm lymphedemas, lymphatic grafts are taken from the patient's thigh and are anastomosed with major lymphatic collectors at the upper arm and lymphatic vessels or lymph nodes at the neck, bypassing the obstruction like a typical vascular bypass $(2,3)$. This method is based on comprehensive basic studies $(3,16,17)$.

Using lymphatic grafts and reconstructing the lympho-vascular system itself, has multiple advantages. Coagulation of lymph is low in contrast to blood (18). Patency rates of lymphatic to lymphatic anastomoses are extremely high, as shown in animal models (17). Lymphatic vessels retain their ability to actively pump lymphatic fluid without innervation (19) and can form anastomoses spontaneously once connected closely to each other (20). Of particular importance is the fact that lymphatic collectors are not merely tubes. With their ability to transport lymph actively, they may act as pumps and are capable of draining lymph from tissues in areas where the peripheral lymphatic collectors in longstanding lymphedema have lost their intrinsic pumping activity. This approach is further supported by the favorable results of other groups utilizing different microsurgical approaches of lymphatic grafting in advanced cases of lymphedema compared to lympho-venous anastomoses or lymph node transplantation (21). A number of early $(3,4)$ and also recent studies (6) including those by other groups have documented the benefit of this procedure (22).

We previously demonstrated that a significant volume reduction can be achieved especially in arm edemas and is sustained in long-term studies with a follow-up period exceeding 10 years (4). Nuclear medicine studies revealed a significant improvement of the lymphatic transport index (5-7,23), which represents the amount of lymphatic fluid transported from an extremity.

Additionally, the donor area can be evaluated by lymphatic scintigraphy. Since all patients underwent a preoperative lymphatic 
scintigraphy of the affected extremity and possible donor site, it was also possible to evaluate the donor site postoperatively. In a consecutive series of 19 patients, no adverse effects or diminished lymphatic transport, as measured by the lymphatic transport index, was seen (24). This result might be due to the fact that we strictly avoid narrow areas of the lymphatic system at the groin or the knee region. Here we only remove lymphatic vessels above the branch point and under the condition that multiple stained lymphatic collectors running within the ventro-medial bundle can be left in place untouched. Long term patency of the lymphatic grafts was demonstrated after surgery using indirect lymphography and MRI lymphography $(7,25,26)$. These studies provide objective data to document the improvement and in some cases normalization of lymphatic transport.

From a perspective of quality of life, not only the volume reduction is important but also the freedom from ongoing treatment involving visible devices and time-consuming treatment sessions. Quality of life was improved significantly when patients who had already experienced improvement by conservative therapy, underwent lymphatic grafting (1). Once again, the improvement was especially important for patients with arm edemas, since elastic stockings and the swollen arm are often obvious in public. Therefore, it is of utmost importance for patients to avoid additional treatments.

With the assistance of liposuction, accumulated fat can be removed sufficiently in a minimally invasive manner. Brorson and colleagues showed that full volume reduction with liposuction alone, was possible if patients are wearing elastic garments continuously $(14,27)$. This result may be fully sufficient for elderly patients and in a cold environment. However, quality of life studies revealed that patients may suffer substantially from the necessity of using these devices and supports (1). We therefore addressed the question whether it is possible to achieve similar results without additional treatment after first improving the lymphatic transport capacity.

Before the first microsurgical intervention, all patients underwent CDT manual lymphatic drainage, continuous compression garment and physical therapy. Most patients utilized CDT for years. The shortest period of CDT was 6 months since during this period transient edemas may disappear spontaneously, and surgery is not recommended in those cases.

After the first microsurgical treatment, elastic support was provided to support the influx of lymph into the anastomoses for the first 6 months. Thereafter, elastic stockings were discontinued and patients monitored closely. Manual lymphatic drainage and compression garments were reinitiated as necessary. Volume measurements where performed using the method according to Kuhnke (11). This method gives a sufficient measurement of volume comparable to water replacement techniques (28).

Our experiences taught us that in moderate lymphedemas with little surplus of fat tissue, adequate results can be achieved by improving and restoring the lymphatic transport capacity alone $(3,8)$. However, many patients with a high volume of fat deposits desire a complete reduction of the excess volume. These patients are therefore candidates for an additional liposuction procedure. The rationale of performing the lymphatic vessel bypass prior to a secondary liposuction procedure is to first correct the causative problem of the lymphedema and restore lymphatic transport.

After the secondary liposuction procedure, a flat-knitted compression garment was applied for $\mathbf{6}$ months and no additional manual lymphatic drainage was applied as outlined above. Since we performed liposuction strictly in parallel to the lymphatic collectors which we had to preserve and spare the area of the microsurgical anastomoses at the proximal upper arm, we did not aim for absolute reduction of arm volumes to normal values. However, overtreatment can occur as illustrated in Figs. $6 a, b$. The critical question was, whether this approach would enable patients having 
suffered such severe lymphedemas to maintain the size of the extremity without additional subsequent treatment.

As described in this report, most patients do not require any subsequent therapy with this sequential approach. Since the liposuction is performed distally to the bypass, potential damage to the bypass is not an important consideration during SLS and liposuction is not performed first. Approaches based on liposuction only are associated with a high recurrence rate of lymphedema when compression garments are discontinued because the underlying problem of lack of lymphatic transport persists. However, lymphatic grafting has a documented effect on improving lymphatic transport and therefore should minimize or avoid the necessity for additional treatment completely, once volume reduction with SLS is achieved. Reduction of additional tissue via SLS also lowers the lymphatic load and improves the balance between the restored lymphatic transport capacity and lymphatic load. Our long-term follow-up studies revealed the freedom from any additional treatment in more than two-thirds of the patients who underwent lymph vessel bypass followed by SLS. This finding suggests that our approach is a highly successful therapeutic strategy to restore form and function of a limb affected by secondary lymphedema without the necessity of ongoing therapy.

\section{CONFLICT OF INTEREST AND DISCLOSURE}

The authors declare no competing financial interests exist.

\section{REFERENCES}

1. Springer, S, M Koller, RG Baumeister, et al: Changes in quality of life of patients with lymphedema after lymphatic vessel transplantation. Lymphology 44 (2011), 65-71.

2. Baumeister, RG, J Seifert, B Wiebecke, et al: Experimental basis and first application of clinical lymph vessel transplantation of secondary lymphedema. World J. Surg. 5 (1981), 401-407.
3. Baumeister, RG, S Siuda: Treatment of lymphedemas by microsurgical lymphatic grafting: What is proved? Plast. Reconstr. Surg. 85 (1990) 64-74; discussion 5-6.

4. Baumeister, RGH, A Frick: Die mikrochirurgische Lymphgefaesstransplantation. Handchir. Mikrochir. Plast. Chir. 35 (2003), 202-209.

5. Kleinhans, E, RG Baumeister, D Hahn, et al: Evaluation of transport kinetics in lymphoscintigraphy: Follow-up study in patients with transplanted lymphatic vessels. Eur. J. Nucl. Med. 10 (1985), 349-352.

6. Weiss, M, RG Baumeister, A Frick, et al: Lymphedema of the upper limb: Evaluation of the functional outcome by dynamic imaging of lymph kinetics after autologous lymph vessel transplantation. Clin. Nucl. Med. 40 (2015), e117-e123.

7. Weiss, M, RG Baumeister, K Hahn: Post-therapeutic lymphedema: Scintigraphy before and after autologous lymph vessel transplantation: 8 years of long-term follow-up. Clin. Nucl. Med. 27 (2002), 788-792.

8. Baumeister, RGH. Reconstructive Lymph Vascular Surgery. Springer, 2017, 21 pp.

9. Frick, A, JN Hoffmann, RG Baumeister, et al: Liposuction technique and lymphatic lesions in lower legs: Anatomic study to reduce risks. Plast. Reconstr. Surg. 103 (1999), 1868-1873; discussion 74-75.

10. Hoffmann, JN, JP Fertmann, RG Baumeister, et al: Tumescent and dry liposuction of lower extremities: Differences in lymph vessel injury. Plast. Reconstr. Surg. 113 (2004), 718-724; discussion 25-26.

11. Kuhnke, S: Volumenbestimmung aus Umfagsmessungen. Folia Angiol. 24 (1976), 228-232.

12. Foeldi, M: Physiologie des Lymphgefaesssystems. Angiologica. 8 (1971), 212-235.

13. Ryan, TJ: Lymphatics and adipose tissue. Clin. Dermatol. 13 (1995), 493-498.

14. Brorson, H, H Svensson: Complete reduction of lymphoedema of the arm by liposuction after breast cancer. Scand. J. Plast. Reconstr. Surg. Hand Surg. 31 (1997), 137-143.

15. Kinmonth JB. The Lymphatics: Surgery, Lymphography, and Diseases of the Chyle and Lymph Systems. Baltimore, Maryland: Edward Arnold; 1982, 428 pp.

16. Baumeister, RGH, J Seifert, B Wiebecke: Transplantation of lymph vessels on rats as well as a first therapeutic application on the experimental lymphedema of the dog. Euro. Surg. Res. 12 (1980), 7.

17. Baumeister, RGH, J Seifert, B Wiebecke: Homologous and autologous experimental lymph vessel transplantation: Initial experience. Int. J. Microsurg. 3 (1981), 19-24. 
18. Yoffey, JM, FC Courtice: Lymphatics, Lymph and the Lymphomyeloid Complex. Academic Press, 1970, 942 pp.

19. McHale, NG: The lymphatic circulation. Irish J. Med. Sci. 161 (1992), 483-486.

20. Danese, C, R Bower, J Howard: Experimental anastomoses of lymphatics. Arch. Surg. 84 (1962), 6-9.

21. Stepniewski, A, SM Weiß, G Felmerer: Microsurgical therapy of lymphedema of the leg - a prospective study. LymphForsch. 23 (2019), 6-15.

22. Felmerer, G, T Sattler, C Lohrmann, et al: Treatment of various secondary lymphedemas by microsurgical lymph vessel transplantation. Microsurgery. 32 (2012), 171-177.

23. Weiss, M, RGH Baumeister, K Hahn: Therapieplanung und Erfolgskontrolle der autologen Lymphgefaesstransplantation mittels nuklearmedizinischer Lymphabflussszintigraphie. Handchir. Mikrochir. Plast. Chir. 35 (2003), 210-215.

24. Weiss, M, RG Baumeister, MJ Zacherl, et al: [Microsurgical Autologous Lymph Vessel Transplantation: Does Harvesting Lymphatic Vessel Grafts Induce Lymphatic Transport Disturbances in the Donor Limb?]. Handchir. Mikrochir. Plast. Chir. 47 (2015), 359-364.
25. Notohamiprodjo, M, M Weiss, RG Baumeister, et al: MR lymphangiography at 3.0 T: Correlation with lymphoscintigraphy. Radiology. 264 (2012), 78-87.

26. Notohamiprodjo, M, RG Baumeister, TF Jakobs, et al: MR-lymphangiography at $\mathbf{3 . 0}$ T--a feasibility study. Eur. Radiol. 19 (2009), 2771-2778.

27. Brorson, H, H Svensson: Liposuction combined with controlled compression therapy reduces arm lymphedema more effectively than controlled compression therapy alone. Plast. Reconstr. Surg. 102 (1998), 1058-1067; discussion 68.

28. Megens, AM, SR Harris, C Kim-Sing, et al: Measurement of upper extremity volume in women after axillary dissection for breast cancer. Arch. Phys. Med. Rehabil. 82 (2001), 1639-1644.

Ruediger G.H. Baumeister, MD, PhD

Professor of Surgery

Ludwig-Maximilians-University of Munich

Drozzaweg 6

81375 Muenchen, Germany

E-mail: baumeister@lymphtransplant.com

Phone: ++49 89583219 